\title{
Frame Alignment Strategies of Brand Globalization from the Perspective of Social Movement Theory
}

\author{
Huang Jing \\ Economics and Management School, Wuhan University, \\ College of Foreign Studies, Jinan University, Guangzhou, P.R.China \\ e-mail: tjinghuang@jnu.edu.cn
}

\begin{abstract}
Drawing from social movement theory in sociology, this article provides a linkage between brand frame alignment strategies and the resonance gaining of global brands in different social and cultural contexts. Based on the literature review and discussion, the author proposes a conceptual brand communication framework and frame alignment strategies which are hypothesized to affect the consumers' evaluations of global brands through resonance in host country markets. The companies from emerging markets can have a positive impact on the consumers' evaluations of global brands through the following two aspects of effort: (1) to set up a brand communication framework, specific methods including amplification strategy, extension strategy and bridging strategy; (2) to communicate with consumers by gaining resonances of credibility resonance, consistency resonance, and commensurability resonance. A grasp of frame alignment resonance dynamics in new markets give managers methods to navigate complex host environments. This study also increases theoretical understanding of the role of frame and rhetoric strategies in brand globalization among the host country markets.
\end{abstract}

Keywords- brand frame alignment strategies; social movement theory; brand globalization; frame alignment resonance; rhetoric strategies

\section{INTRODUCTION}

More and more attention has been focused on globalization and markets development. However, most of the current existing researches focus on material strategy level, and ignore the importance of rhetorical strategies for brand globalization, especially for emerging market companies.

Developing and managing brand image is an important part of a firm's global marketing program. A clearly defined brand image enables consumers to identify the needs satisfied by the brand. Many firms face challenges of gaining resonance in foreign markets. Although brand communication strategies have been discussed in the global context, the relationship between global brand image management and communication strategies have not been well understood. This article introduces global brand frame alignment strategies as an important brand communication approach of the resonance gaining process in host country markets.

Developing a resonance-based image strategy provides the foundation for creating new markets in host countries. Firms are successful to the degree to which they can successfully navigate the environment. Kotler notes that breaking ground in new markets, both domestic and international, requires megamarketing, "the strategically coordinated application of economic, psychological, political, and public relations skills to gain the cooperation of a number of parties in order to enter and operate in a given market" ${ }^{,[1]}$. Some marketing scholars have outlined the need for a sociological approach that accounts for the influence of multiple stakeholders in the process of market creation ${ }^{[1]}$. The rhetorical strategy for brand globalization is also essential for the companies, especially for those from the emerging markets.

On the rhetorical level, articulation of frame is closely related with the brand resonance. Frame articulation is the explanatory mechanisms in the process of interpretation.

\section{FRAME RESONANCE}

The concept of frame resonance is relevant to the issue of the effectiveness or mobilizing potency of proffered framings ${ }^{[1]}$. Some framings seem to be effective while others do not. Today, social sciences have analyzed the concept of frame frequently. Frames can vary in terms of the degree of resonance. The articulators frame or arrange meaning to relevant events and brands in ways that are intended to mobilize potential consumers and stakeholders to support them. There are several dimensions of resonance: credibility resonance, consistency resonance and commensurability resonance.

\section{A. Credibility}

Credibility is the fit between the framing and events in the world ${ }^{[1]}$. Credibility is a necessary condition for enhancing the resonance of claims. The claims of the frame should be empirically verified and "real" to the consumers and stakeholders. The greater the number of evidence the frame, the more empirically believable the claimed events and brands, and the more credible the framing and resonance. Therefore, the brand should build speicific and believable frames to gain the consumers' resoanance.

\section{B. Consistency}

Consistency refers to the congruency between an articulated beliefs, claims, and actions [1]. It is a wellestablished fact that speakers who are regarded as more credible are generally more persuasive. Thus, the greater 
the status and perceived expertise of the frame articulator, and the more plausible and resonant the framings or claims.

\section{Commensurability}

Commensurability can contribute to a frame's salience ${ }^{\text {[1] }}$. It has to do with how essential the values, ideas and cultures associated with the brand frames are the lives of the targets of communication. The closer the framings, the greater their salience, and the greater the probability of mobilization. The framings should resonant with the personal, everyday practice of the audience. The framings cannot be too abstract and distant from the lives and experiences of the targets.

Previous research has examined three types of resonance. Credibility resonance rests on the selfinterested calculations of an organization's most immediate audiences. Often, this immediacy involves direct exchanges between organization and audience; however, it also can involve broader social and cultural interdependencies, in which organizational action effectively affects the audience's well-being. Consistency resonance and commensurability resonance refers to the degree to which a brand frame can be understood, integrated and classified in existing social and cultural environment in host countries.

Resonance building of a foreign firm is generally a proactive enterprise, because managers have advance knowledge of their plans and of the need for resonance gaining. The author proposed three clusters of resonancebuilding dimensions for firms from emerging markets: (a) efforts to conform to the experience of preexisting audiences within the organization's current environment, (b) efforts to select the right consistent frames in pursuit of an audience that will support current practices, and (c) efforts to manipulate environmental structure by conforming to the existing culture and creating new beliefs and new audiences. All three efforts involve complex mixtures of concrete organizational change and persuasive organizational communication; however, they clearly fall along a continuum from relatively passive conformity to relatively active articulation; and little is known about the rhetorical strategies used to frame the global brands in host country markets.

\section{Frame ALIGNMENT StRATEGIES}

\section{A. Frame Analysis}

The role of language in communication has been discussed by some scholars ${ }^{[1]}$. Scholars have shown how issues can be framed in specific ways to advance or resist the legitimation of particular phenomena, decisions, practices, or changes in them. Different accounts can provide radically different understandings of issues, and thus lead to resonance and recognition. Closely related, scholars have shown how impression management is a central part of legitimation. Lately, scholars have then singled out specific elements in rhetorical justification and identified rhetorical strategies ${ }^{[1]}$.

Frame analysis has been used extensively in sociology to study changes in political and cultural discourses over time ${ }^{[1]}$. Frames are "individual cognitive structures ... that orient and guide interpretation of individual experience". They enable a person to "selectively punctuate and encode objects, situations, events, and experience within one's present and past environment" "11]. To accomplish this punctuation and encoding, they "draw from the supporting ideas and norms of ideologies, but are understood as more specific cognitive structures advanced by social actors to shape interpretation and understanding of specific issues". Frames are the linguistic tools by which social actors attempt to manipulate legitimacy over time.

In sociology, not only has the framing concept been applied most to cognitive psychology, linguistics, discourse analysis, communication, media and political science, but interest in framing processes in relation to the operation of marketing has animated an increasing amount of conceptual and empirical scholarship. Scholars have suggested that the framing of an object or concept in the media can have a profound effect on its legitimacy ${ }^{[1]}$.

\section{B. Frame Alignment Strategies}

Ashlee Humphreys offered a new perspective on the creation of markets by viewing it as a process of legitimation, and assessed the deployment of framing strategies by managers, and examined company press releases and interviews with executives and reanalyzed quotations from industry executives in the newspaper data set ${ }^{[1]}$. Ashlee Humphreys found that casino proponents employ the framing strategies of amplification, extension, and bridging at different points in the legitimation process. Stakeholders use specific frames to shape the perceived legitimacy of an industry and these frames are effective in markets ${ }^{[1]}$.

What are the best strategies for becoming the accepted, dominant industry in a crowded competitive landscape? Research on social movements might be helpful to conceptualize the ways industries and firms compete for legitimacy through cultural frames ${ }^{[1]}$.

Such research can be applied here to assess the ways that framing strategies taken by executives to legitimate the international firms in host countries. I extend this logic to argue that any internationalization process in a new market requires the same strategic efforts. Global brands can benefit from its framing strategic implications for gaining foreign consumers' frame resonance in host countries.

Some rhetorical strategies have been outlined by previous research: amplification, extension, bridging ${ }^{11}$. Amplification is the idealization, embellishment, clarification, or invigoration of existing values or beliefs. In the case of the global brand marketing, amplification occurs when the company's representatives emphasize the advantages of the products and service, the history and culture of the companies. This strategy idealizes the practice, making it congruent with the customers' value in host countries. Extension is a framing strategy in which proponents enlarge the initial concept, "extending it beyond its primary interests to include issues and concerns that are presumed to be of importance to potential adherents". Extension in the case of global brands takes the form of enlarging the initial concept of a product or service. The strategy of extension can be used to appeal to multiple stakeholders, like consumers, investors, and local partners. Bridging is the linking of two or more ideologically congruent but structurally unconnected frames regarding a particular issue or problem. Bridging in the case of global 
brands takes the form of linking two cultures or concepts. For example, changing consuming habits in the host country markets, like coffee consumption and life styles.

To understand the market creation of global brands in host countries, this article draws from theory of social movement in sociology. The deployment of frame alignment strategies by the executives can be found from firms' press release and interviews with managers and reanalyzed quotations in the newspaper and on main comprehensive financial websites.

\section{CONSUMER IDENTITY}

With the development of globalization, many consumers are becoming members of multiple sociocultural milieus and hold multiple social identities, which in turn influence their judgments and decisions ${ }^{[1]}$. Arnett suggests that consumers today exhibit characteristics of both local and global identities ${ }^{[1]}$. A local identity means that consumers feel they belong to their local community and identify with local ways of life, whereas a global identity means that consumers feel they belong to the global community and identify with a global lifestyle. A study by Zhang and Khare showed that identifying consumers' identity as global or local is key to understanding consumers' attitudes toward global versus local products ${ }^{[1]}$. Thus, brand managers need to understand whether customers feel they are part of a global or local community to manage the positioning of global versus local products.

How will local and global identities affect consumers' brand resonance evaluations? Studies of social judgment found the way individuals respond to cultural cues depends on their cultural identity structure. Individuals with global identity tend to assimilate to cultural cues, yet individuals with local identity contrast to these cues.

Therefore, the author proposes that the consumers' global/local identity will moderate the effect of frame alignment strategies.

\section{RESEARCH PROPOSITION}

Based on the above literature review and discussion, this article proposes the following propositions (refer with: Fig. 1):

Proposition 1: Amplification frame alignment strategy will have a positive impact on the brand resonance.

Proposition 2: Extension frame alignment strategy will have a positive impact on the brand resonance.

Proposition 3: Bridging frame alignment strategy will have a positive impact on the brand resonance.

Proposition 4: Amplification frame alignment strategy will have a positive impact on the consumers' evaluation of global brands.

Proposition 5: Extension frame alignment strategy will have a positive impact on the consumers' evaluation of global brands.

Proposition 6: Bridging frame alignment strategy will have a positive impact on the consumers' evaluation of global brands.

Proposition 7: The global brand resonance will mediate between the frame alignment strategies and evaluation of global brands.
Proposition 8: The consumer global/local identity will moderate between the frame alignment strategies and brand resonance.



Figure 1. Framework of frame alignment strategies, resonance and evaluation of brand.

\section{CONCLUSIONS}

This article attempts to integrate the research findings of social movement theory and marketing communication strategies in the context of global marketing. A grasp of frame resonance dynamics in new markets give managers methods to navigate complex host environments. Frame alignment strategies are analyzed as brand communication strategies for global brands to gain resonance and enter new foreign markets. The companies from emerging markets can have a positive impact on the consumers' evaluation of global brands through the following two aspects of effort: (1) to set up a brand communication framework, specific methods including amplification strategy, extension strategy and bridging strategy; (2) to communicate with consumers by gaining frame resonances of credibility resonance, consistency resonance, and commensurability resonance.

\section{REFERENCES}

[1] Kotler, Philip. Megamarketing[J]. Harvard Business Review, 1986, 64 (2):117-25.

[2] Giesler, Markus. How Doppelganger Brand Images Influence the Market Creation Process: Lon-gitudinal Insights from the Rise of Botox Cosmetic [J]. Journal of Marketing, 2012,76 (6),55-68.

[3] Benford, Robert D. and David A. Snow. Framing Processes and Social Movements: An Overview and Assessment[J]. Annual Review of Sociology, 2000, 26 (1):611-40. 
[4] Matthes, Jörg and Matthias Kohring. The Content Analysis of Media Frames: Toward Improving Reliability and Validity[J]. Journal of Communications, 2008, 58 (2), 258-79.

[5] Jones, Candace and Reut Livne-Tarandach. Designing a Frame: Rhetorical Strategies of Architects[J]. Journal of Organizational Behavior, 2008, 29 (8), 1075-1099.

[6] Johnston, Josee and Shyon Baumann. Democracy Versus Distinction: A Study of Omnivorousness in Gourmet Food Writing[J]. American Journal of Sociology, 2007, 113 (1), 165204.

[7] Humphreys, Ashlee. Semiotic Structure and the Legitimation of Consumption Practices: The Case of Casino Gambling[J]. Journal of Consumer Research, 2010, 37 (3):490-510.
[8] Suchman, Mark C. Managing Legitimacy: Strategic and Institutional Approaches[J]. Academy of Management Review, 1995, 20 (3), 571-611.

[9] Humphreys, Ashlee. Megamarketing: The Crea-tion of Markets as a Social Process[J]. Journal of Marketing, 2010, 74 (2):1-19.

[10] Arnett, Jeffrey J. The Psychology of Globalization[J]. American Psychologist, 2002, 57 (10), 774-83.

[11] Zhang and Khare.The impact of accessible identities on the evaluation of global versus local products[J]. Journal of Consumer Research, 2009, 36(10) , 524-537 